Handbuch der Gaschromatographie

Leibnitz · Struppe 


\section{Unter Mitarbeit von}

Hans-Karl Bothe, Leipzig · Claus-Eberhardt Döring, Leuna - Wilhelm Ecknig, Berlin - Werner Engewald, Leipzig - Günter Eppert, Leuna - Dietrich Estel, Halle-Neustadt - Jürgen Fischer, Böhlen - Gerhard Gnauck, Berlin - Dieter Habisch, Berlin · Manfred Hofmann, Leipzig · Jürgen Köller, Berlin · Thomas Kratzsch, Leipzig · Günter Kraus, Halle · Jörg Kühne, Erlangen - Walter Leipnitz, Halle-Neustadt - Wolfgang Markgraf, Königs
Wusterhausen - Klaus Metzner, Halle-Neustadt . Manfred Mohnke, Leipzig - Dankwart Obst, Berlin - Arpád Pethö, Hannover · Peter Popp, Leipzig . Jürgen Pörschmann, Leipzig - Wolfgang Rödel, Bergholz-Rehbrücke · Dieter Rodewald, Leipzig · Harald Rotzsche, Radebeul/Dresden - Helmut Schön, Berlin - Ernst Stottmeister, Bad Elster, Hans Georg Struppe, Leipzig - Wolfgang Walther, Berlin 


\section{Handbuch}

der

Gaschromatographie

Herausgegeben von

\section{Prof. Dr.- Ing. Dr. h. c. Eberhardt Leibnitz, Berlin}

\section{Dr. Hans Georg Struppe, Leipzig}

3., überarbeitete und stark erweiterte Auflage

Mit 500 Abbildungen und 143 Tabellen

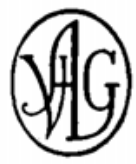

AKADEMISCHE VERLAGSGESELLSCHAFT GEEST \& PORTIG K.-G. LEIPZIG 1984 
(c) Akademische Verlagsgesellschaft Geest \& Portig K.-G., Leipzig 1984

3. Auflage

VLN 276-105/11/84 - LSV 1235

Lektor: Dr. rer. nat. Ingrid Lischke

Printed in the German Democratic Republic

Gesamtherstellung:

INTERDRUCK Graphischer Großbetrieb Leipzig,

Betrieb der ausgezeichneten Qualitătsarbeit, III/18/97

Bestell-Nr. 6696402 